\title{
The $t$-test, resource pooling, and psi: why the minimum sample size counts towards theoretical progress in behavioral science
}

\author{
${ }^{1}$ Erich H. Witte \& ${ }^{2 *}$ Frank Zenker \\ ${ }^{1}$ University of Hamburg, Institute for Psychology, Von-Melle-Park 5, 20146 \\ Hamburg, Germany; ORCID: 0000-0002-9446-3211 \\ ${ }^{2}$ Boğaziçi University, Department of Philosophy, 34342 Bebek, Istanbul, Turkey; \\ ORCID: 0000-0001-7173-7964; *corresponding author: fzenker@gmail.com
}

Abstract: A theoretical construct that subsumes an empirical phenomenon should rest on statistically significant test-results with high replication probably. To statistically establish such test-results, behavioral science publications typically rely on a $t$-test, and researchers typically operate under limited data collection resources. To publish more test-results, or to publish an individual result sooner, questionable strategies are commonly used to reduce data collection cost. One strategy is to increase the $\beta$-error rate from 0.05 to 0.20 ; a second strategy is to treat the control group as a constant, collapsing a two sample $t$-test into a one sample $t$-test. Both strategies happen to underlie Bem's (2011) rightly controversial results on human precognition ("psi hypothesis" [Journal of Personality and Social Psychology, 100, 3, 407-425]). Since both strategies undermine theoretical research in behavioral science, their ubiquity partially explains the rarity of well-corroborated theoretical constructs there. We advocate collaboration between individual labs as a viable route to enabling theoretical research by collecting a large enough sample jointly.

Keywords: Fisher; Neyman-Pearson; psi research; power; $t$-test, theory construction 


\title{
The $t$-test, resource pooling, and psi: why the minimum sample size counts towards theoretical progress in behavioral science
}

\author{
Erich H. Witte \& Frank Zenker
}

\section{Introduction}

In behavioral science, the probably most frequently used statistical test to compare the independent means in a controlled experimental study is the $t$-test. To reduce the minimum sample size $\left(N_{\mathrm{MIN}}\right)$ for a $t$-test, two strategies are commonly applied to save data collection cost. The first strategy lowers test-power from $(1-\beta)=0.95$ to $(1-\beta)=0.80$, lowering the probability that the original test-result is independently replicated. The second strategy collapses a Neyman Pearson-test into a Fisher-test, making test-results useless to develop an empirically adequate theoretical construct that is more informative than a non-random effect. Both strategies happen to underlie Bem's (2011) rightly controversial and misleading results on human precognition ("psi hypothesis").

Researchers seemingly deploy both strategies to publish test-results although a study's actual sample $(N)$ fails to meet $N_{\text {MIN }}$, itself an element of Neyman-Pearson test-theory (NPTT) (Neyman \& Pearson, 1967). We argue that, in order to develop a theoretical construct, a theoretician needs well-powered test-results, $(1-\beta) \geq 0.95$. Conversely, spending less than what NPTT identifies as necessary data collection cost is guaranteed to undermine theoretical efforts. This claim extends beyond a $t$ test, to NPTT-based tests that compare multiple means, variances, correlations, or frequencies (Cohen, 1977; ${ }^{2}$ 1988).

That both strategies are normal in behavioral science today goes along with research here focusing not on the development of theoretical constructs, but on the discovery of non-random test-results in individual studies. This focus partially explains the replication crisis in behavioral science, whose root cause is the inability to independently repeat a non-random test-result (Pashler \& Wagenmakers, 2012; Klein et al., 2018; Camerer et al., 2018). To support the development of informative theoretical constructs (more precise than a two-sided non-random hypothesis or even a one-sided directional non-random hypothesis), we advocate that behavioral scientists should bear the cost of data collection collectively. 


\section{Induction vs. corroboration}

With rare exception, behavioral science today regularly operates with small samples. In psychology, for instance, the typical sample size is $N<30$ (Bakker, van Dijk \& Wicherts, 2012). But a "small $N$-study" can discover a statistically significant effect, only if the empirically observed effect is large. Yet, behavioral science studies typically find small to medium size empirical effects, the actual $N$ regularly being much smaller than $N_{\text {MIN }}$. This implies that published statistically significant testresults tend to be underpowered, leaving their independent replication improbable.

A statistically significant but underpowered test-result generally is a poor instance of an effect that a theoretician would seek to subsume under a theoretical construct. If a good theory predicts a true effect, then a statistically significant but underpowered effect is what a theoretician should ignore. Conversely, only wellpowered statistically significant test-result warrant the effort of developing a theoretical construct (Witte \& Zenker, 2018). Therefore, if $N<N_{\mathrm{MIN}}$, then researchers avoid what from a theoretical point of view are necessary data collection cost.

To fully appreciate this point, it helps to contrast induction with deduction as two central modes of statistical reasoning. Researchers invariably collect data to induce an empirically observed effect size, often estimating it as Cohen's $d$, the difference between the means in experimental and control group, divided by the standard deviation, $d=\left(m_{1}-m_{2}\right) / s$, where $0 \leq|d| \leq \infty$. ${ }^{1}$ By convention, $d=0.01$ is considered a very small effect, $d=0.20$ is small, $d=0.50$ medium, $d=0.80$ large, $d=1.20$ very large; and some call $d=2.0$ huge. Crucially, inducing $d$ in ways a theoretician can take seriously requires that empirical data satisfy two criteria:

\section{(C1) Statistical significance criterion}

\footnotetext{
${ }^{1}$ Preacher and Kelley (2012) report that $d$ is probably the most widely used effect size measure today, while Schaefer et al. (2019) report that it probably is $r$. Another common measure is Hedges' $g$. Regardless which measure is most common, one can safely transform $d$ into $r$ or $g$, if experimental and control groups have similar base rates, because this maximizes a binary variable's variance (McGrath \& Meyer, 2006). Compared to $d$, only extremely diverging base rates (e.g., 0.95 vs. 0.05 ) would reduce $r$ artificially. For other transformations, see Schulze (2004, 30ff).
} 
On the assumption that the (random) $\mathrm{H}_{0}$, itself postulating $d=0$, is the best-fitting description of data, $\mathrm{D}$, the observed empirical effect, $d=x$, is sufficiently improbable $(p)$, where the statistical significance threshold is conventionally set to 0.05 . In form: $p\left(\mathrm{D}, \mathrm{H}_{0(d=0)}\right)<0.05$.

\section{(C2) Replicability criterion}

Assuming that $\mathrm{H}_{1(d=x)}$ is the true value, replicating the point-specified effect $d=x$ in $n e w$ data is sufficiently probable, i.e., $p\left(\mathrm{D}, \mathrm{H}_{1}\right)>(1-\beta)$, where the conventional replication threshold is set to $(1-\beta)=0.80$.

Since ' $p\left(\mathrm{D}, \mathrm{H}_{x}\right)$ ' denotes neither a conditional nor a joint probability, it conveys a meaning that is distinct from $p\left(\mathrm{D} \mid \mathrm{H}_{x}\right)$ or $p\left(\mathrm{D} \& \mathrm{H}_{x}\right)$. If $d$ is transformed into a statistic, e.g., a $t$-value, then $p\left(\mathrm{D}, \mathrm{H}_{\mathrm{x}}\right)$ denotes the probability of observing this statistic, or more extreme values of it. As a natural language expression for $p\left(\mathrm{D}, \mathrm{H}_{\mathrm{x}}\right)$, this suggests: 'the probability of $\mathrm{D}$ in view of $\mathrm{H}_{\mathrm{x}}$ '.

Fisher's (1956) approach to statistical inference had merely introduce the $p$ value, which suffices to estimate $p\left(\mathrm{D}, \mathrm{H}_{0}\right)$. The $p$-value thus succeeds in addressing C1. But Fisher's approach ignores $\mathrm{H}_{1}$, and so fails to address $\mathrm{C} 2$. Fisher's approach therefore offers neither an effect size measure, nor a replication probability measure (Mayo, 1996).

These two measures were only introduced in NPTT, which estimates $p\left(\mathrm{D}, \mathrm{H}_{0}\right)$ and $p\left(\mathrm{D}, \mathrm{H}_{1}\right)$ by drawing on:

(i) the $\alpha$-error rate (aka $p$-value): the probability of mistakenly accepting that observed data deviate significantly from $\mathrm{H}_{0}$ (false positive error rate);

(ii) the $\beta$-error rate: the probability of mistakenly accepting that observed data deviate significantly from $\mathrm{H}_{1}$ in the direction of $\mathrm{H}_{0}$ (false negative error rate); respectively the derived magnitude ( $1-\beta$-error), i.e., the probability that data replicate if $\mathrm{H}_{1}$ is true (aka. test-power);

(iii) the effect size, $d$ : the theoretically predicted or the empirically observed (non-random) causal effect, or correlation, between independent and dependent variables;

(iv) the minimum sample size, $N_{\mathrm{MIN}}$, given both $d$ and the $\alpha$ - and $\beta$-error rates. 
While origins, uses, and interpretations of the $p$-value and the $\alpha$-error rate differ, and although both concepts are never equivalent, the $p$-value nevertheless relates to the $\alpha$-error rate. In fact, both concepts can be combined to arrive at a binary decision. Originating in NPTT, the $\alpha$-error rate represents the theoretical long-run probability of committing a Type I-error (false positive error rate). The $\alpha$ error rate thus serves as a decision criterion when choosing between the two sides of a simple hypothesis. NPTT requires two such hypotheses, at least one of which, the $\mathrm{H}_{1}$, differs from a data-description because, in NPTT, the $\mathrm{H}_{1}$ derives from a theory.

Originating in Fisher's approach, by contrast, the $p$-value represents the empirical probability of observing data at least as extreme as data in fact sampled, if $\mathrm{H}_{0}$ is true. The $p$-value thus describes an empirical test-result in view of a single hypothesis, typically a random version of the $\mathrm{H}_{0}$. Since Fisher's approach offers merely $\mathrm{H}_{0}$ and the $p$-value, but no $\mathrm{H}_{1}$-hypothesis, his approach can only measure whether data deviate from a random distribution $\left(\mathrm{H}_{0}\right)$. The $p$-value thus is useful to decide that something non-random has occurred, yet without implying a substantial hypothesis as to what has occurred. Given these constraints, the commonly used $t$ test with two error-probabilities $(\alpha, \beta)$ is therefore perfectly unintended in Fisher statistics; it becomes possible only under the constraints of NPTT.

A researcher seeking to decide whether, given data, one should pursue the $\mathrm{H}_{0}$ or the $\mathrm{H}_{1}$ would consider the probability of a mistaken decision. The $p$-value bases this probability on data (objective), while the $\alpha$-error-rate bases that probability on the researcher's personal expected error-rate (subjective). Since the decision should be based on data, a researcher would typically set the expected personal error-rate no higher than the $p$-value serving as the statistical significance criterion. In practice, therefore, the differences between both concepts are effectively "hidden."

Thus, although the $p$-value and the $\alpha$-error rate are non-equivalent concepts, one can meaningfully treat the empirically observed $p$-value as if it were the theoretical $\alpha$-error, and thus arrive at an empirically-based binary decision whether to accept or reject a hypothesis. Moreover, since NPTT has only three degrees of freedom, fixing any three of $\alpha, \beta, d$ and NMIN lets the fourth magnitude "fall out." Hence, given specified $\alpha$ - and $\beta$-error rates, as well as a point-specific value for $d$ motivated by theoretical reasoning, NPTT deduces $\mathrm{N}_{\text {MIN. This feature recommends }}$ NPTT as a planning tool to estimate $N_{\mathrm{MIN}}$, and thus determine necessary data 
collection cost, using an a priori test-power analysis (Cohen, 1977; 19882) as follows:

$$
N_{\mathrm{MIN}}=2\left[z_{(1-\alpha)}+z_{(1-\beta)}\right]^{2} / d^{2}
$$

Here, $z$ is the value of $d$ for which the area under a standard normal distribution has a probability density that corresponds to the $\alpha$ - and $\beta$-error rates. Given $\alpha=\beta=0.05$ and $d=0.50$, for instance, $\mathrm{N}_{\mathrm{MIN}}=2[1.65+1.65]^{2} / 0.50^{2}=87.12$, or approximately $N_{\mathrm{MIN}}=88$.

Interpreted as normative magnitudes, the $\alpha$ - and $\beta$-error rates state error-rates that researchers have determined by convention. The effect size, by contrast, can be determined in but one of three ways: (i) again by convention (e.g., following Cohen, as very small, small, medium, or large), (ii) by induction, as an observed $d$-value, or (iii) by deduction based on theoretical considerations, as a theoretical $d$-value. Thus, if data collection cost are calculated before data collection starts, then $N_{\text {MIN }}$ is the only material resource a researcher has to determine.

Since the induction $v s$. deduction-distinction reflects the basic purpose of research, it also constrains the shape of the appropriate formal support-measure. It is by induction that the probability measure $p\left(\mathrm{D}, \mathrm{H}_{0}\right)$ estimates the probability of an observed test-result in view of the data-distribution postulated by $\mathrm{H}_{0}$. Here, 'D' denotes an interval comprising empirically observed data, as well as more extreme possible data. To corroborate a hypothesis, by contrast, is to measure the extent to which D—now interpreted as theory-deduced point-statistic — supports $\mathrm{H}_{1}$ or $\mathrm{H}_{0}$. As a distinct measure, corroboration requires the likelihood ratio (LR): $L\left(\mathrm{H}_{1} \mid \mathrm{D}\right) / L\left(\mathrm{H}_{0} \mid \mathrm{D}\right)=p\left(\mathrm{H}_{1}\right) \times p\left(\mathrm{D}, \mathrm{H}_{1}\right) / p\left(\mathrm{H}_{0}\right) \times p\left(\mathrm{D}, \mathrm{H}_{0}\right)$, a likelihood being a probability multiplied by a positive constant (Edwards, Lindman \& Savage, 1963; Edwards, 1972). (Decisive reason against using the Bayes factor as a corroboration measure are given in Witte and Zenker (2017b), and Krefeld-Schwalb, Zenker, and Witte (2018).)

Unlike in the inductive case, 'D' now denotes a point-specified observation, for instance the point-difference between the expected mean effect in experimental and control group. In view of criteria $\mathrm{C} 1$ and $\mathrm{C} 2$, then, only data that are statistically significant and well-powered can offer sufficient empirical support to corroborate a 
theoretical hypothesis. In behavioral science, however, researchers show little interest in inducing effect sizes that enable a statistical hypothesis corroboration. In fact, a recent survey of some 200 meta-analyses based on almost 8,000 publications in psychology journals reports median test-power of $(1-\beta)=0.36$ (Stanley, Carter \& Doucouliagos, 2018). This analysis also notes that only some 8\% (!) of studies here achieve $(1-\beta)=0.80$. Bakker et al. (2012) report similar results.

The main telos of research thus appears to be the publication of statistically significant test-results under minimal data collection cost, results that address $\mathrm{C} 1$ but fail to address $\mathrm{C} 2$. That this must play out negatively for theory construction is what we now show in the context of a $t$-test.

\section{The $t$-test}

To plan a proper control group study, where $N \geq N_{\text {MIN }}$, one must stipulate not only $\alpha$, $\beta$, and $d$, but also two data distributions. For a small sample $(N<30)$ and an estimated standard deviation, the distributions are known as $t$-distributions (Fig. 1). Although a $t$-distribution can only approximates observed data, statistical inferences are possible by acting as if observed data mirrored a normally distributed variable with a corresponding small sample standard deviation, $s$. For example, we may hypothesize $d_{\mathrm{H} 0}=0$ as the mean value in the control group, and $d_{\mathrm{H} 1}=0.50$ in the experimental group. When transforming $d_{\mathrm{H} 1}$ into the $t$-distribution's non-centrality parameter $\delta=d \times \sqrt{ }(N / 2)$, and provided the sample size is the same in both groups $\left(N_{1}=N_{2}\right)$, we find that $\delta$ depends only on $N .^{2}$ Given $N_{1}=N_{2}=88$, it thus follows that $d_{\mathrm{H} 0}=0$ yields $\delta=d \times \sqrt{ }(\mathrm{N} / 2)=0$, whereas the largest probability density for $d_{\mathrm{H} 1}$ is found at the point $\delta=3.32$ (Fig. 1.) So, beyond the ordinate $t=1.65$ (aka 'critical $t$ '), the probability density for $\mathrm{H}_{1}$ exceeds that for $\mathrm{H}_{0}$. For any observed data point exceeding critical $t$, therefore, the probability of falsely rejecting $\mathrm{H}_{0}$ is smaller than $\alpha=0.05$.

\footnotetext{
${ }^{2}$ If $N_{1} \neq N_{2}$, a useful sample must be neither too small, nor too large. Compared to a $t$-test where $N_{1}=N_{2}$, the harmonic, geometric, and arithmetic mean deliver an everincreasing value, and thus estimate the actual sample size ever less conservatively.
} 


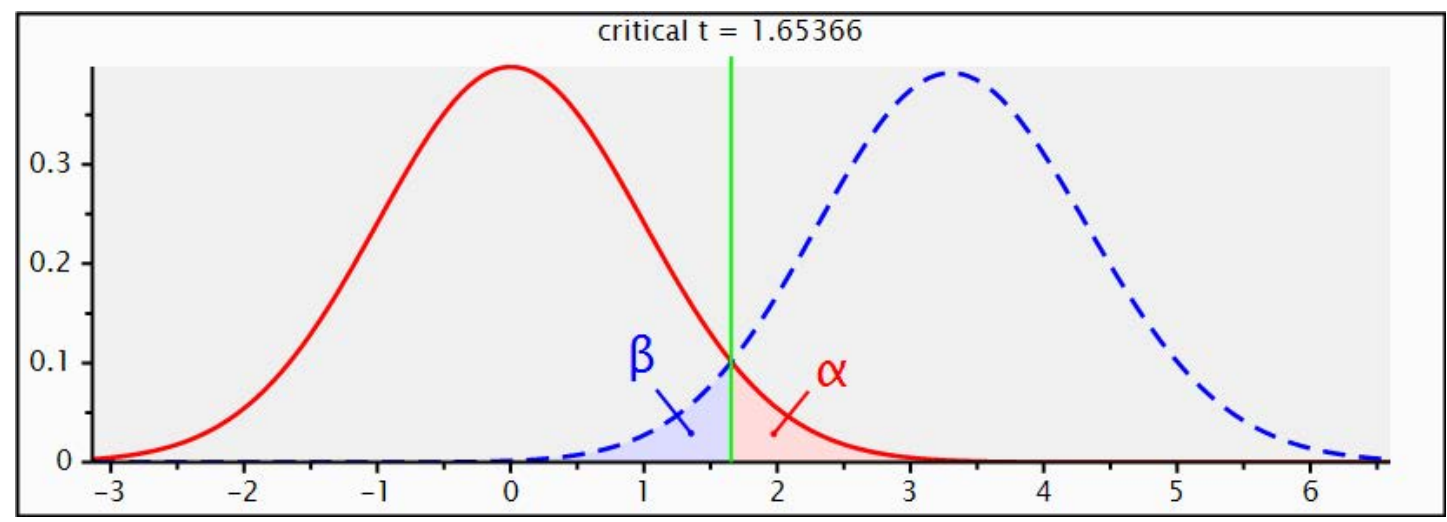

Fig. 1 One-sided, two sample $t$-test for $d=0.50$ given $N_{1}=N_{2}=88$ and $\alpha=\beta=0.05$. The hypothetical $\mathrm{H}_{0}$-distribution (solid line) is centered on $\delta=0$; the observed $\mathrm{H}_{1}$-distribution (dashed line) is centered on $\delta=3.32$. The abscissa ( $x$-axis) states the $t$-value given $N$; the ordinate ( $y$-axis) states the probability density for a given $t$-value. Up to critical $t=1.65366$, each shaded area $(\alpha, \beta)$ covers $5 \%$ of the other distribution. (Figure generated with G*Power; Faul, Erdfelder, Lang \& Buchner, 2007).

To ensure that a study meets $N_{\text {MIN }}$, computer software readily facilitates an a priori test-power analysis (e.g., G*Power; Faul, Erdfelder, Lang \& Buchner, 2007). But if median test-power is in fact no larger than about $(1-\beta)=0.36$ (Stanley, Carter \& Doucouliagos, 2018), then the majority of published behavioral science studies that report statistically significant results would have determined $\mathrm{N}$ either at liberty-e.g., by stopping data collection once a test-result is statistically significant, which Bayarri et al. (2016) rightly call "cheating" —or would have determined N strictly according to available resources (e.g., the number of students in a class).

This suggests that the aim of publishing an empirical test-result regularly dominates the aim of securing sufficient test-power. Of course, if a researcher focuses only on the observed effect in the first place, but ignores the theoretically expected effect, then test-power cannot be computed other than post-hoc. Absent any theoretical considerations, however, Bakan's (1966) well-known objection applies. Given $N \geq 60.000$, even a very small effect can become statistically significant by splitting the sample randomly. So, absent theoretical considerations, statistical significance is guaranteed to be a trivial criterion once $N=60.000$. 
Irrespective of the specific effect size a statistically significant study reports, to the extent that the $\alpha$ - and $\beta$-error rates both increase, the effect size becomes ever less useful for constructing a theory that predicts a true effect. Ceteris paribus, if $\mathrm{H}_{1(d=x)}$ were true, then as the $\beta$-error-rate increases, the probability that a statistically significant effect replicates decreases. Conversely, ceteris paribus, if $\alpha=\beta$, then $d=x$ becomes more useful for the same purpose as $\mathrm{N}$ increases, because both error-rates decrease. (Formally, decreasing the error rates increases the non-centrality parameter $\delta$, which reduces the $t$-distributions' overlap in Fig. 1.) So, if $\alpha=\beta$, then already in case a prediction postulates nothing stronger than a directional deviation from random, a statistically significant effect's use-value for theory construction stands or falls with the $\beta$-error-rate.

Cohen $\left(1977 ;{ }^{2} 1988\right)$ had recommended asymmetric error rates $(\alpha \neq \beta)$, viz. $\alpha=0.05, \beta=0.20$. He stated that, given $\alpha=0.05$ as the antecedently accepted error rate, "[w]hen the investigator has no other basis for setting the desired power value, the value 0.80 is used" (Cohen, 1977, 56). Rather than motivating the value of 0.80 independently, however, Cohen accepted — without further argument - that it is more important to avoid false positive test-results than to avoid false negative ones (ibid.). Despite being text-book example of circular reasoning, his recommendation became so entrenched that $\alpha=0.05$ and $\beta=0.20$ are widely accepted today as the "right" error-rates.

Asymmetric error rates imply that researchers weigh the risk of falsely accepting that an effect is statistically significant $(\alpha)$ against the risk of failing to replicate a true non-random effect $(\beta)$ (Witte, 1994; Witte \& Kaufman, 1997). Given $N_{1}=N_{2}=50\left(N_{\text {TOTAL }}=100\right)$, for instance, if $d=0.50$, then $\alpha=0.05$ one-sided yields $(1-\beta)=0.80$, whereas $\alpha=0.05$ two-sided yields $(1-\beta)=0.70$, and $\alpha=0.01$ one-sided even only yields $(1-\beta)=0.56$, i.e., close to chance level. Yet, to meaningfully develop a theoretical construct, a theoretician needs error rates of $\alpha=\beta<0.05$, or lower. What else should a theoretical construct subsume if not the true effect?

Worse yet, researchers in fact pay lip service to Cohen's recommendation, while undermining it regularly. As publishing such test-results renders them of very limited use for theory development, we proceed to critique two common strategies meant to reduce necessary data collection cost. That both strategies happen to 
underlie Bem's (2011) results on human precognition (aka "psi-effect") entails strong reasons to doubt these results.

\section{Two cost reduction strategies}

\subsection{From $\mathrm{N}_{M I N}=176$ to $\mathrm{N}_{M I N}=27$}

Since the behavioral science literature typically reports small to medium sized effects (Cafri, Kromrey, \& Brannick, 2010), and given theoretically motivated errorrates of $\alpha=\beta<0.05$, collecting all of $N_{\mathrm{MIN}}$ can quickly generate massive data collection cost, as shown in Table 1 for the significance level $\alpha=0.05$.

Table 1: The estimated minimum sample size $\left(N_{\text {MIN }}\right)$ for a two sample $t$ test as a function of test-power $(1-\beta)$ and effect size, $d$, given $\alpha=0.05$

\begin{tabular}{ccccc}
\hline & \multicolumn{4}{c}{$d$} \\
\cline { 2 - 5 }$(1-\beta)$ & 0.01 & 0.20 & 0.50 & 0.80 \\
& very small & small & medium & large \\
\hline 0.40 & 38726 & 97 & 15 & 6 \\
0.50 & 54111 & 135 & 22 & 8 \\
0.80 & 123651 & 309 & 49 & 19 \\
0.95 & 216443 & 541 & 88 & 34 \\
\hline
\end{tabular}

Necessary data collection cost thus are what many researchers today appear to shun. For test-results in empirical psychology, for instance, the largest behavioral science field, meta-analyses have consistently estimated median test-power as $0.35 \leq(1-\beta) \leq 0.50$ (Cohen, 1962; Bakker, van Dijk \& Wicherts, 2012; Stanley et al., 2018). Given publication bias, moreover, the behavioral science literature typically underrepresents the actual proportion of statistically non-significant results (Cafri, Kromrey, \& Brannick, 2010; Francis, 2012; Sedlmeier \& Gigerenzer, 1989). Although preregistration can eliminate this bias, even the recent well-powered preregistered replication studies could replicate the original effect size in only $25 \%$ of all cases (Klein et al., 2018; Schäfer \& Schwartz, 2019).

To understand how underpowered test-results arise, let us critically consider two strategies that seemingly help researchers "save" up to $85 \%$ of necessary data collection cost. We already saw that a one-sided two sample $t$-test for a medium 
effect $(d=0.50)$ under $\alpha=\beta=0.05$ requires $N_{\mathrm{MIN}}=N_{1}=N_{2}=88\left(\mathrm{~N}_{\mathrm{TOTAL}}=176\right)$. The first strategy maintains $\alpha=0.05$ but increases $\beta=0.05$ - the $\beta$-error value that theoretically considerations warrant - by a factor of 4 , to $\beta=0.20$. This reduces $N_{\text {MIN }}$ by some $42 \%$ to $N_{1}=N_{2}=51(102 \approx 0.58 \times 176)$. Thus, rather than obtaining only one in twenty false positive statistically significant test-results that probably fail to replicate, one obtains one in five such results.

Underlying the second strategy is the assumption that random influences among data cancel out mutually. A given causal influence or correlation of size $x$ in one direction would thus be matched by a corresponding influence of size $x$ in the opposite direction. This assumption underlies Bem's (2011) psi-effect research (aka precognition), allowing Bem to treat the hypothesis his experiment shall falsify, the $\mathrm{H}_{0}$, as postulating the constant zero, or when expressed as a binary variable as postulating a 50\% chance of a fair coin landing heads up. Experimental participants' average empirical reactions that fail to display a psi-effect are thus assumed to describe a random distribution.

This assumption, or so the following quote shows, allows Bem to avoid an actual (empirical) control group, and thus allows him to deploy a one sample $t$-test against a theoretically postulated constant random effect in an imaginary control group (Bem et al., 2011; Bem, Tressoldi, Rabeyron, \& Duggan, 2016).

"I set 100 as the minimum number [aka $\mathrm{N}_{\mathrm{MIN}}$ ] of participants/sessions for each of the experiments reported in this article because most effect sizes $\left(d_{\mathrm{emp}}\right)$ reported in the psi literature range between $\left[d_{\mathrm{emp}}=\right] 0.20$ and 0.30. If [one sets the theoretical effect size to the point-value] $d_{\text {theo }}=0.25$ and [the sample to] $\mathrm{N}=100$, the power to detect an effect as significant at $[\alpha=] 0.05$ by a one-tail, one sample $t$-test is $[(1-\beta)=] 0.80 . "(\mathrm{Bem}, 2011$, 409 , fn. 1 , notation adapted)

As a consequence, data collection cost are massively reduced. A (one-sided) two sample $t$-test given $d=0.25, \alpha=0.05$, and $(1-\beta)=0.80$, requires $N_{1}+N_{2}=\mathrm{N}_{\mathrm{MIN}}=398$. Yet, given the same values of $d, \alpha$, and $\beta$, a (one-sided) one sample $t$-test only requires the $\mathrm{N}_{\mathrm{MIN}}=100$ that Bem mentions. And, a one sample $t$-test given a medium-sized effect of $d_{\text {theo }}=0.50, \alpha=0.05$, and $(1-\beta)=0.80$ even only requires 
$\mathrm{N} 2=\mathrm{N}_{\mathrm{MIN}}=27$, close to the median sample size of published studies in psychology, $\mathrm{N}=24$ (Wetzels et al., 2011). This makes a "median-study" with $N_{\mathrm{MIN}}=24$ nearly equivalent to a one sample $t$-test given $\alpha=0.05,(1-\beta)=0.80$, and $d=0.50$ (Fig. 2). If data collection cost are a linear function of $\mathrm{N}_{\mathrm{MIN}}$, which often is safe to assume, then compared to $\mathrm{N}_{\mathrm{MIN}}=398$, this saves some $75 \%$ of cost $(398 \times 0.25 \approx 100)$.

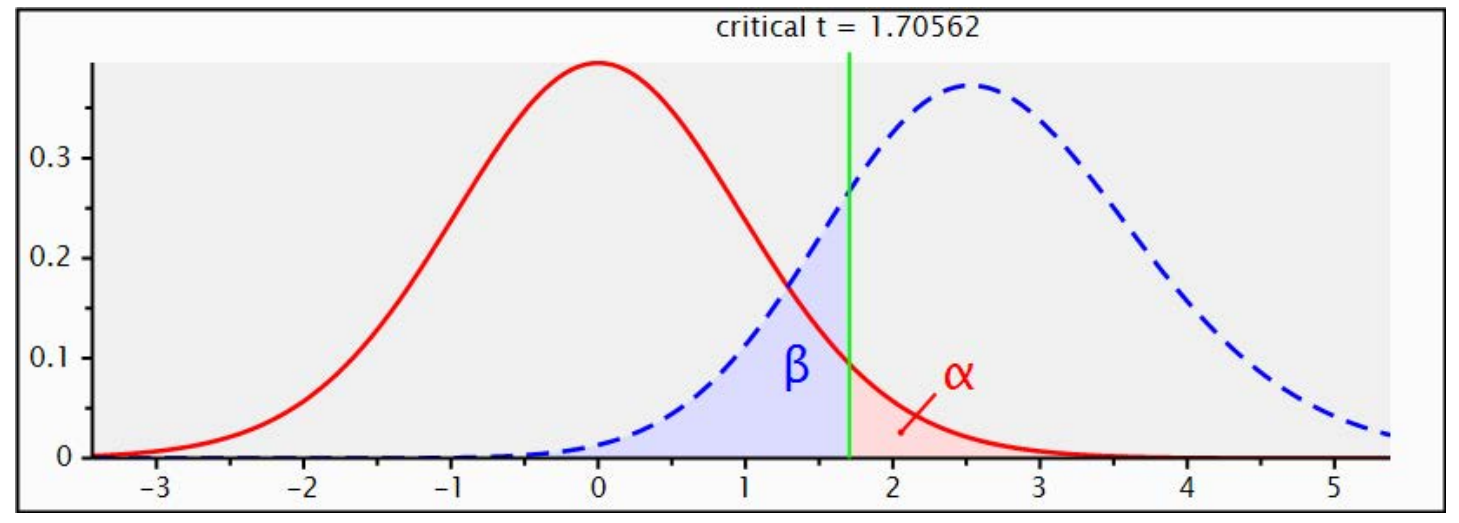

Fig. 2 One sample $t$-test for the difference from a constant of zero, given $\alpha=0.05, \beta=0.20, d=0.50, N=27, t=1.70562$. Despite the term 'constant', one must employ two distributions rather than one to calculate test-power, including a second, theoretically predicted distribution centered on a theoretically motivated constant. (Compare labels in Fig. 1.)

The successive application of both strategies - increasing the $\beta$-error-rate from 0.05 to 0.20 ; treating the control group as a constant - thus results in $25 \%$ of $N_{\text {MIN. }}$ But it risks masking Bem's own inference strategy. By citing $\alpha, \beta, d$ and $N_{\text {MIN }}$, after all, Bem presents the inference strategy as if it were based on NPTT. Empirical measurements, however, occur only in the experimental group. (There is no actual control group.) Bem's point- $\mathrm{H}_{0}$ thus effectively predicts his own empirical setting's unknown influences. Such influences, however, cannot be measured systematically because they arise from factors besides the focal experimental effect. In fact, the control-group strategy was developed precisely because uncontrollable influences cannot be controlled. So, a genuine NPTT-test must use an actual control group. 
Unlike the theoretically expected effect size, only the empirically observed effect size can describe a specific experimental condition's sample size, as well as that sample's specific standard deviation. In a two sample $t$-test, as we saw, the $d$-value reports the difference between the independent mean effect sizes in the experimental group and control group, $m_{1}-m_{2}$, divided by the standard deviation, $s$. Since independent samples correlate to degree zero $\left(r_{12}=0\right)$, one can derive $s$ by adding the square root of the variances from each sample: $s=s_{(x 1-x 2)}$, where $s_{(x 1-x 2)}^{2}=s_{(\mathrm{x} 1)}+s^{2}{ }_{(\mathrm{x} 2)}-$ $2 \times r_{12} \times s_{(\mathrm{x} 1)} \times s_{(\mathrm{x} 2)}$. So, if $s^{2}{ }_{(x 1)}=s^{2}(x 2)$, then $d=\left(m_{1}-m_{2}\right) / \sqrt{ } 2 s$. By normalizing the standard deviation to $s=1$, one obtains $d=\left(m_{1}-m_{2}\right) / \sqrt{ } 2=\left(m_{1}-m_{2}\right) / 1.41$.

Compared to a two sample $t$-test, then, if only a single sample's standard deviation is used to define the effect size, then it is by the value 1.41 that the standard deviation of a one sample $t$-test necessarily decreases. After all, the $t$-test's one sample variant sheds $m_{1}$, retaining only $m_{2}-c$, the difference between the mean of the experimental group's data distribution and a constant random effect, $c$. Yet, the constant random effect $c$ is a point-specified effect, rather than a distributed effect. To a constant effect, however, one cannot meaningfully associate a standard deviation. In $d^{*}=\left(m_{2}-c\right) / s_{(x 1-x 2)}$, therefore, the independently estimated value of $s_{(x 1-x 2)}$ must be lower than in $d=\left(m_{1}-m_{2}\right) / s_{(x 1-x 2)}$.

As explained above, the observed effect size is an inductive concept, while the theoretically expected effect size is a deductive concept. Test-power, however, is meaningfully associated only to the latter concept, serving to plan experiments with $N_{\text {MIN. }}$ Based on NPTT, such planning necessitates associating two hypotheses and two error-distributions with each theoretically postulated mean. Reducing this to one error-distribution implicitly increases $d$, because the inference is now based on only one distributions, where $\alpha$ - and $\beta$-error are undefined (see Figs. 1 and 2). In brief, if a statistical inference shall be based on NPTT (featuring defined $\alpha$ - and $\beta$-errors), then the numerator will be increased, because two distributions with an estimated standard deviation, $s$, are needed. In all other cases, the inference simply is not "according to the art."

Specifically, $d$ relates to the $t$-value as: $t=d / \sqrt{ } 2 \cdot s$. A one sample $t$-test's $t$ value is therefore more sensitive to empirical data than a two sample $t$-test, namely by the factor 1.41 , above. For instance, $d=0.50$ given two samples would increase to $d^{*}=0.705$ given one sample. Since test-power depends on $d$, it follows that, if one 
holds $\mathrm{N}$ constant, then a one sample $t$-test has greater test-power than a two sample $t$-test. Put differently, if test-power is constant, then $N_{\text {MIN }}$ decreases. This explains why a one sample $t$-test incurs lower data collection cost than a two sample $t$-test.

According to Cohen (1977), the shift from two samples to one is mandatory. The increase in effect size between $d$ and $d^{*}$ shall "compensate for [his own testpower] tables' assumption of double error variance" (ibid., 46). Cohen motivates this assumption by having simplified $s_{(x 1-x 2)}$ to $s_{(x 2)}$. The constant $c$ itself, Cohen "conceive[s] as the mean of a normal distribution whose standard deviation is also $s$ " (ibid., 46). Thus, "values of power are sought in the power tables by means of $d^{*} \times \sqrt{ } 2$ " (ibid., 47; notation adapted). We consider this an illegitimate transformation of a Fisher-test into an NPTT-test, which must feature two error-prone hypotheses.

\subsection{A one sample t-test is a Fisher-test}

Having assumed a medium effect $(d=0.50)$, we saw how the second strategy lowers $N_{\text {MIN }}$ for a two sample $t$-test by some $85 \%(186 \times 0.15=27)$, while incurring the assumption that $\mathrm{H}_{0}$ can be modeled without an actual control group, i.e., without an empirical data distribution. This assumption is problematic, because if one now fails to obtain $d$ as the difference between two means of a statistical $t$-distribution, and so fails to obtain a test-power value. Yet, without a test-power value, one cannot deploy a genuine NPTT $t$-test. One has rather altogether abandoned this statistical concept.

For both the experimental and the control group, moreover, the standard deviations are normally established empirically, from data. A theory, by contrast, predicts $d$ as a point-specific difference between means. But a reasonable theoretician would not go as far as to predict the exclusively empirical magnitude that a point-specific standard deviation is.

Even if the standard deviation in the experimental group is identical to that in the control group, there remains a non-zero probability that the experimental group's $t$-value $\left(\mathrm{H}_{1}=d\right)$ is an element of the $t$-distribution centered on $\mathrm{H}_{0}=c$ (Figs. 1 and 2). Call this probability the $p$-value. But to properly deploy an NPTT-test, the constant $c$ fails to represent $\mathrm{H}_{0}$ properly. (One would additionally have to center a random distribution on $c$; otherwise the $\beta$-error is undefined.) Since Bem's $t$-test uses the improper representation of the $\mathrm{H}_{0}$ that a "distributed constant" $c$ is, his $t$-test treats experimental group data as if they originated from the random distribution 
representing the group mean, $c$. The test-result, however, is meant to report the probability to which data deviate statistically significantly from the distribution placed around the constant $c$. But Bem's $t$-test can evaluate data only in view of $\mathrm{H}_{0}=c$. The empirical decision criterion, therefore, is not the $\alpha$-error-rate, as defined in NPTT, but the empirically derived $p$-value.

This entails that Bem deploys not an NPTT-test, but a Fisher-test. After all, the attempt to interpret a one sample $t$-test as an NPPT-test would incur the unacceptable assumption that the control group's mean can be specified as the theoretical point-value $c$, without any variation. But point-specification is unrealistic because any experimental condition is subject to uncontrolled empirical influences. That, indeed, is why researchers should use an actual control group, to which participants are randomly assigned vis-à-vis an experimental group.

As a Fisher-test, a one sample $t$-test requires three values: the random distribution around $c$, the observed mean of the experimental group, $m_{2}$, and the estimated standard deviation placed around $c$, the latter obtained from the observed values of the experimental group, $s_{(\mathrm{x} 2)}$. The standardized mean difference-measure thus is $d=m_{2}-c / s_{(x 2)}$. Transformed into a statistic, it becomes $t=d_{\mathrm{emp}} \times \sqrt{ } N / 2$. So, if $d^{*}=0.50$, then given $N=27$, one finds $t=1.84$. Under a random distribution, therefore, if $t=1.84$, then a mere $3 \%$ of all $t$-values are more extreme than the empirically observed $t$-value. But nowhere is there a $\mathrm{H}_{1}$-hypothesis, and thus no test-power. All Bem's $t$-test allows to obtain is the $p$-value that, given a random distribution, potential empirical test-results are the same, or yet more extreme than, an observed result. This is all that a one sample $t$-test reports.

Starting with an NPTT-test under conventionally accepted error rates, but then to reducing $\mathrm{N}_{\text {MIN }}$ to $15 \%$ while continuing to present it as a NPTT $t$-test under constant error rates, appears highly questionable. Given $N_{1}=N_{2}=27\left(\mathrm{~N}_{\mathrm{TOTAL}}=54\right)$, $\alpha=0.05$, and $d=0.50$, even a proper two (independent) sample $t$-test would at most have test-power of $(1-\beta)=0.57$. Close to chance, this nearly implies speculative decision-making. At any rate, already Cohen's own (lenient) standard $(1-\beta)=0.80$ entails $N_{\mathrm{MIN}}=N_{1}=N_{2}=51\left(\mathrm{~N}_{\mathrm{TOTAL}}=102\right)$. 


\subsection{Double testing}

A proper $t$-test for $d=0.50$ (one-sided) given $\alpha=\beta=0.05$ thus needs $N_{\text {MIN }}=N_{1}=N_{2}=88$ $\left(\mathrm{N}_{\text {TOTAL }}=176\right)$. Given the typical effect size range of published observed effects in behavioral science $(0.10<d<0.50)$, as the $d$-value shrinks, $N_{\text {MIN }}$ quickly reaches beyond the data collection resources that individual research groups commands. This suggests that resource restrictions are a major reason for $N$ to stay below $N_{\text {MIN }}$, while letting a $t$-test seem a genuine NPTT-test (with conventionally accepted error rates).

Even if resource restrictions keep researchers from collecting all of $N_{\mathrm{MIN}}$, the one strategy that NPTT offers to legitimately reduce $N_{\text {MIN }}$ is double testing. 'Double testing' refers to testing the same participants in random order, in both the control and the experimental group condition, and then calculating for each subject the difference between both conditions (repeated measurement design). Double testing obviously creates a dependent sample. And, compared to how the measurement error spreads in two independent samples $\left(N_{\text {TOTAL }}=176\right)$, one can arguably reduce $s_{(x 1-x 2)}$ legitimately — and thereby increase the effect size — only if participants' responses in both groups are sufficiently correlated, e.g., to degree $r_{12}=0.50$.

Notice that the following holds: $s_{(x 1-x 2)}^{2}=s^{2}(\mathrm{x} 1)+s_{(\mathrm{x} 2)}{ }^{2}-2 \times r_{12} \times s_{(\mathrm{x} 1)} \times s_{(\mathrm{x} 2)}$. Given independent samples (which correlate to degree $r_{12}=0$ ), therefore, the product $2 \times r_{12} \times s_{(\mathrm{x} 1)} \times s_{(\mathrm{x} 2)}$ goes to zero. Generally, as the standard deviation of two groups, $s_{(x 1-x 2)}$, converges to that of a single group, $s_{(\mathrm{x})}$, the variance of the mean of the differences between both groups continuously grows smaller. If $s_{\mathrm{x} 1}=s_{\mathrm{X} 2}=1$, for instance, then given $r_{12}=0.50$, one finds $s^{2}{ }_{(x 1-x 2)}=s^{2}{ }_{(x 1)}=1$. So, on formal grounds, the study of experimental participants' individual differences in behavior using two groups makes an empirical test-condition statistically comparatively more sensitive that when using but one group. This because the formal model is now less errorprone.

Whether the psychological processes in focus are thought to be identical or even influenced by double testing, any reduction of $N_{\mathrm{MIN}}$ using double testing thus requires the necessary assumption that the value of $r_{12}$ is positive. (Absent contrary evidence, a compromise between not knowing the true correlation and one's resource restrictions may be the initial assumption that $r_{12}=0.50$.) Compared to a two independent sample $t$-test, lowering the error variance by means of double testing 
increases the effect size, and thereby lowers $N_{\mathrm{MIN}}$, and so decreases data collection cost. Given $\alpha=\beta=0.05$, for instance, a matched pairs $t$-test for $d=0.50$ with dependent samples requires $N_{\text {MIN }}=N_{1}=N_{2}=45$. Deploying the test twice yields 90 data points yet requires only 45 participants. This legitimate way of reducing the minimum sample thus lowers $\mathrm{N}_{\mathrm{MIN}}$ from 176 to 45 , or to some $26 \%$.

On this background, we turn to resource pooling as a way for labs to reach

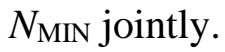

\section{Resource pooling}

\subsection{Resource pooling is possible and meaningful}

A literature review would show the ubiquity of both strategies critiqued in Sect 5 . The strategies' negative consequences for theoretical knowledge, of course, may be widely unknown. Alternatively, these negative consequences, though known, may for the following reasons seem acceptable.

The first reason is practical, citing a research group's lack of sufficient resources to individually meet $N_{\mathrm{MIN}}$, as well as obstacles encountered when seeking to increase $N$ to $N_{\text {MIN }}$ by pooling several labs' resources (e.g., lack of experience, infrastructure, or peer-support). The second reason is motivational, stating that statistically significant, well-powered test-results are a goal that actual resource restrictions simply make unachievable. The third reason cites the impetus to avoid the hypocrisy of desiring what one should want (significant, well-powered testresults), but because of insufficient resources also cannot want.

The cogency of the third reason stands or falls with that of the second reason. As for that second reason, since no law of nature keeps from coordinating resources, several labs can in principle perform the same study under similar conditions to

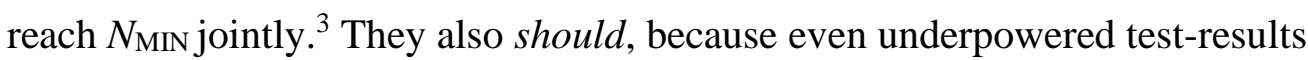

\footnotetext{
${ }^{3}$ See Landy et al. (2020), who report the replication efforts of 15 research teams investigating five social-psychological hypotheses with more than 15.000 experimental participants. They show that the typically large unexplained heterogeneity in results plausibly relates to researchers' subjective choices in designing experimental stimuli. The authors observe, "one way to reduce the role of subjective researcher choices in research outcomes may be to more fully flesh out the underlying theory at the outset" (ibid., 22).
} 
remain theoretically useful to corroborate/falsify a hypothesis if one aggregates their likelihood ratios. Simulation results show that, if the corroboration threshold is sensitive to both error-rates — as holds for $\mathrm{LR}_{\mathrm{H} 1 / \mathrm{H} 0}>(1-\beta) / \alpha(\mathrm{Wald}, 1947)$ - then loglikelihood addition combines underpowered test-results safely (Krefeld-Schwalb, Witte \& Zenker, 2018). For exemplary analyses of combined test-results, see Witte and Zenker (2016) on macro-social stress (Chou, Parmar \& Galinsky, 2016) and Witte and Zenker (2017a) on ego-depletion (Hagger \& Chatzisarantis, 2016). Related proposals on how to limit the publication of underpowered data include Nosek and Bar-Anan (2012), and Nosek, Spies and Motyl (2012). Resource pooling thus compensates fully for an individual lab's limited resources. The second and third reasons thus are non-cogent.

The first reason exclusively cites practical obstacles, which often require innovative solutions to old problems (e.g., how to share academic credit fairly; Rehg, 2009). These practical obstacles, however, certainly cannot be overcome by sticking to the approaches of Fisher or Neyman-Pearson, because their statistical concepts only enable decisions for an individual study. In fact, a method to combine test-results of a series of studies had long remained absent (Birnbaum, 1954). To our best knowledge, the only fair and firm combined statistical test for a series of studies is log-likelihood-ratio addition (Krefeld-Schwalb, Witte \& Zenker, 2018; Witte \& Zenker, 2017b).

\subsection{Research diversity is a weak con-reason}

The one reason against resource pooling cites research diversity (Schönbrodt \& Wagenmakers, 2018). Given constant resources, if labs pool individual resources to achieve sufficient test-power, ceteris paribus, then each lab can "discover" only a comparatively smaller number of statistically significant effects. Yet, assigning value to diverse but underpowered statistically significant test-results, as opposed to less diverse but well-powered significant test-results, does not only subordinate theoretical progress (Witte \& Strohmeier, 2013). An overly strong focus on research diversity without aggregation and subsequent theory development indeed kills theory. After all, the aggregation of diverse yet dissimilar experimental test-results is theoretically as pointless as the comparison of apples and oranges is proverbial (cf. Landy et al., 2020). Not pointless, by contrast, is a differentiation (with respect to 
the same theory) of empirical test-results obtained under dissimilar experimental conditions, But this already implies undertaking at least initial efforts in theory construction. In brief, research diversity can be at most as important as obtaining well-powered significant test-results.

\subsection{Resource pooling is necessary}

Few researchers today manage resource pooling within an integrated research program in service of theoretical goals, even if several recent publications report preregistered replications that look as if an integrated research program were pursued (e.g., Hagger \& Chatzisarantis, 2016; Landy, et al., 2020). Although preregistered replications aggregate the effect sizes of individual studies, no construct is specified as a point-alternative hypothesis. Researchers thus take a first step towards analyzing test-results of similar experimental conditions from different labs, but leave the full theoretical potential unused (Witte \& Zenker, 2017a).

If the theoretical motivation for resource pooling were better understood, and properly incentivized, then training and infrastructure would probably develop accordingly. Test-power would then complement the $p$-value, both in job interviews and on review checklists. And, as journals predominantly published well-powered significant test-results, with the use of likelihood-ratios (rather than probability measures) becoming normal, too, substantial hypothesis verifications could inform theoretical research (Witte \& Zenker, 2017b).

Instead, what researchers in behavioral science do today is seek to replicate originally underpowered effects at a test-power level of around $(1-\beta)=0.80$, the very value Cohen recommended (e.g., Cova et al., 2018). Counter-intuitively, however, once resource pooling leads to test-results under $\alpha=\beta=0.01$, for instance, then the typically effort-intensive replication attempts undertaken today would become superfluous (Witte \& Zenker, 2018). In terms of statistical test-power, after all, independently replicating the statistically significant (non-zero) observed effect size of an already well-powered original study $(\alpha=\beta=0.05)$ yields merely a warrant to restate confidence in the originally observed effect. But unless the error-rates are yet further decreased $(\alpha=\beta<<0.05)$, independently replicating this original effect would fail to offer a warrant to increase confidence in the originally observed effect. 
This is so because the joint replication probability of $n$ independent studies (aka 'aggregate test power') is the product of these studies' test-power, or $(1-\beta)^{n}$. If a first successful replication yields $(1-\beta)=0.80 \times 0.80=0.64$, for instance, then already the second successful replication yields only $(1-\beta)=0.512$. But this is too close to chance-level to use this observed effect size in theory construction. Unlike loglikelihood aggregation paired with $\mathrm{LR}_{\mathrm{H} 1 / \mathrm{H} 0}>(1-\beta) / \alpha$ as the corroboration threshold (Krefeld-Schwalb, Witte \& Zenker, 2018), therefore, to determine whether a testresult is informative for theory construction, aggregate test-power simply is a misleading formal measure.

\subsection{Inadequate samples sizes incur negative consequences}

Hiring decisions regularly assign great importance to a job candidate's number of a publications, using it as a proxy measure for research productivity. Gervais, Jewell, Najle, and $\mathrm{Ng}$ (2015) operationalize research productivity as the sum of two sample control group studies $\left(N_{1}, N_{2}\right)$ that two hypothetical candidates publish over five years. Ceteris paribus, given $d=0.40$ and $N_{1}=N_{2}=100$, the candidate whose studies consistently achieve $(1-\beta)=0.95$ can in principle publish 21 studies with statistically significant results, compared to 33 studies if a candidate makes do with $N_{1}=N_{2}=25$. Given $\alpha=0.05$ (one-sided), however, $N_{1}=N_{2}=25$ implies $(1-\beta)=0.40$, which is below chance level. Even $\mathrm{N}_{1}=\mathrm{N}_{2}=100$ yields only $(1-\beta)=0.88$. Thus, avoiding an erroneous discovery of a non-random effect under $\alpha=0.05$ would thus be evaluated as being 2.4 times more important than avoiding an erroneous rejection of a true alternative hypothesis $(\beta=0.12=0.05 \times 2.4)$.

From a theoretical viewpoint, this evaluation is unreasonable. An under-sized sample, after all, necessarily leaves vague what $\alpha=\beta=0.05$, or even $\alpha=\beta=0.01$, would instead turn into the "well-hardened" (Lakatos, 1978) empirical knowledge of an effect size, knowledge that a theoretician needs to develop a theoretical construct. Indeed, if we assume a medium effect $(d=0.50)$ as typical, then most published effects in behavioral science publications would be underpowered. Behavioral science would thus generally underestimate the negative theoretical consequences of conducting underpowered studies. Indeed, “[r] unning underpowered studies $[\ldots]$ might constitute a type of performance-enhancing design that inflates an individual researcher's productivity while having deleterious consequences for the collective 
enterprise of science" (Gervais et al., 2015, 848). Though a hiring committee would reportedly reverse their decision upon learning that little of theoretical value speaks for underpowered test-results (Gervais et al., 2015), inadequate samples size clearly incur negative consequences for theory construction.

\section{Discussion}

As several recent guidelines make clear, reliable scientific knowledge requires independently replicated test-results (e.g., "Reproducibility and Replicability in Science" (NAS, 2019)). Replicable results in turn are more important than novel results, and the ability to theoretically predict replicable results is more important yet. The replication crisis in behavioral science, as we saw, can be explained as a consequence of typically low $(1-\beta)$-error-rates, that is, an overly low probability of replicating an original test-result. The root cause of this crisis, we submit, is a wide misunderstanding or misapplication of statistical methods in contexts of limited data collection resources, because reducing $N$ to a value smaller than $N_{\text {MIN }}$ must be "paid" for in terms of test-power. And, both strategies critiqued here-lowering the $(1-\beta)$-error-rate from 0.95 to 0.80 ; treating the control group mean as a constantare typical examples of questionable research practices to reduce $N_{\text {MIN }}$.

As most behavioral science research today is data-driven, not only do many researchers misunderstand/misapply the best statistical methods (Gigerenzer, 2018), they also tend to over-interpret or overstate a test-result's significance. An indicator of this is the verbal transition — without warrant - from 'is (statistically) significant' to 'is (scientifically) important'. A related issue arises from assuming "exactly zero effect of site, experimenter, stimuli, task, instructions, and every other factor except subject" (Yarkoni, 2020), resulting in a mismatch between general verbal statements of theoretical hypotheses and their statistical expressions (aka 'generalizability crisis'). Finally, the preference for novel results in behavioral science has long kept from appreciating the full value of replication research (Witte \& Strohmeier, 2013; Ioannidis, 2005; Pasher \& Wagenmakers, 2012; Open Science Collaboration, 2015). In fact, these causes were already discussed in the 1970s (e.g., Meehl, 1967; 1978).

A theoretically progressive behavioral science requires the ability to derive point-specific predictions from theories that subsume well-replicated experimental effects, thereby contributing to explaining, predicting, and intervening upon these effects. Indeed, the practical value of theoretical knowledge rests squarely on 
successful interventions. Though the philosophy of science has long recognized induction and deduction as distinct modes of reasoning, behavioral science regularly conflates these modes, here taking it for granted that learning from already observed ('old") data would provide a substitute for a theory-based prediction of new data. Particularly Bayesian methods embrace this idea.

But predictions derive from theoretical knowledge, whereas induction can practically succeed without any theoretical accountability. While induction describes the process of arriving at a parameter estimate given old data (aka 'retrodiction'), a point-specific version of this estimate is what a theoretical construct must predict for a specific empirical condition, and then test this prediction against new data. A confirmation by data, therefore, can be nothing other than a confirmation of a theory-derived point-prediction. Conversely, theories failing to point-predict cannot be confirmed by data. Indeed, absent point-predicting theories that retrodict old data and predict new data, theoretical progress is hard to define and hard to achieve.

Since the construction of empirically adequate theoretical constructs is impossible without collecting much larger samples (that guaranteed acceptable replication probability), collaboration between labs in the form of resource pooling remains a promising and viable route to avoid the negative consequences for theoretical knowledge in behavioral science. Even Nobel prize winner Daniel Kahneman admitted, in 2017 (Schimmack et al., 2017), that his bestselling “Thinking fast and slow" (Kahneman, 2011) relied on too many non-replicated empirical effects. Of course, it does not help that "published papers in top psychology, economics, and general interest journals that fail to replicate are cited more than those that replicate" (Serra-Garcia \& Gneezy, 2021, 1).

\section{Conclusion}

The functional dependence between the $\alpha$ - and $\beta$-error-rates, $d$, and $N_{\text {MIN }}$ allows researchers to plan empirical studies with sufficiently large samples to achieve testresults under $\alpha=\beta=0.05$, test-results that alone can warrant the effort of subsuming the corresponding observations under a theoretical construct. Presently, however, these very error-rates would filter out the bulk of published test-results in behavioral science as broadly useless for theory construction.

We have here critiqued two common strategies-lowering the $(1-\beta)$-errorrate from 0.95 to 0.80 ; treating the control group mean as a constant—as typical 
examples of questionable research practices to reduce $N_{\text {MIN }}$. We saw that, even if $\alpha=\beta=0.05$, a proper two sample $t$-test of the means in experimental and control group always requires a point-specification of the $\mathrm{H}_{0}$ and the $\mathrm{H}_{1}$. Provided that $r_{12}$ is positive, moreover, double testing is the only admissible strategy to reduce $N_{\text {MIN }}$.

A one sample $t$-test, by contrast, collapses a two sample Neyman-Person test into a Fisher test, which merely reports the $p$-value of data in view of $\mathrm{H}_{0}$. Although the $p$-value can have heuristic value, a Fisher test cannot estimate test-power, hence cannot statistically corroborate/falsify a theoretical hypothesis, so fails to inform theory construction. As we have argued, collaboration between individual labs in the form of resource pooling remains a viable route to increasing the sample sizes in behavioral science.

Acknowledgements: For comments on earlier versions, we thank audiences at Beijing Normal University, PRC, the Universities of Gdansk and Poznan, Poland, Hamburg, Germany, Lund, Sweden, and Waterloo, Canada. FZ acknowledges funding from the European Union (CA17132) and TUBITAK (118C257).

Declarations: Both authors declare that they have no conflicts of interest with respect to the authorship or the publication of this article. EHW wrote a first draft of the manuscript, both authors edited it, and both approved the final submitted version. All values were calculated using G*Power software (Faul et al., 2007).

\section{References}

Bakan, D. (1966). The test of significance in psychological research. Psychological Bulletin, 66, 423-437

Baker, M. (2015). First results from psychology's largest reproducibility test. Nature: https://doi.org/10.1038/nature.2015.17433

Bakker, M., Dijk, van A., \& Wicherts, J. M. (2012). The rules of the game called psychological science. Perspectives on Psychological Science, 7, 543-554. https://doi.org/10.1177/1745691612459060

Bayarri, M. J., Benjamin, D. J., Berger, J. O., \& Sellke, T. M. (2016). Rejection odds and rejection ratios: A proposal for statistical practice in testing hypotheses. Journal of Mathematical Psychology, 72, 90-103. https://doi.org/10.1016/j.jmp.2015.12.007 
Bem, D.J. (2011). Feeling the future: Experimental evidence for anomalous retroactive influences on cognition and affect. Journal of Personality and Social Psychology, 100, 3, 407-425. https://doi.org/10.1037/a0021524

Bem, D. J., Utts, J., \& Johnson, W. O. (2011). Must psychologists change the way they analyze their data? Journal of Personality and Social Psychology, 101(4), 716-719. http://dx.doi.org/10.1037/a0024777

Bem, D., Tressoldi, P., Rabeyron, Th., \& Duggan, M. (2016). Feeling the future: A meta-analysis of 90 experiments on the anticipation of random future events [version 2; referees: 2 approved] F1000 Research 2016, 4,188. https://doi.org/10.12688/f1000research.7177.2

Birnbaum, A. (1954). Combining independent tests of significance. Journal of American Statistical Association, 49, 559-574.

Cafri, G., Kromrey, J. D., \& Brannick, M. T. (2010). A meta-meta-analysis: Empirical review of statistical power, type I error rates, effect sizes, and model selection of meta-analyses published in psychology. Multivariate Behavioral Research, 45, 239-270. http://dx.doi.org/10.1080/00273171003680187

Camerer, C. F., Dreber, A., Holzmeister, F., Ho, T. H., Huber, J., Johannesson, M., ... \& Altmejd, A. (2018). Evaluating the replicability of social science experiments in Nature and Science between 2010 and 2015. Nature Human Behaviour, 2(9), 637-644. https://doi.org/10.1038/s41562-018-0399-Z

Cohen, J. (1962). The statistical power analysis for the behavioral sciences: a review. J. Abnorm. Soc. Psychol., 65, 145-153. http://dx.doi.org/10.1016/B978-0-12-179060-8.50012-8

Cohen, J. (1977, rev. ed., 1988). Statistical power analysis for the behavioral sciences. London: Academic Press.

Cova, F., Strickland, B., Abatista, A. G. F., Allard, A., Andow, J., Attie, M., ..., \& Zhou, X. (2018, April 21). Estimating the Reproducibility of Experimental Philosophy. Review of Philosophy and Psychology (online first). https://doi.org/10.1007/s13164-018-0400-9

Chou, E. Y., Parmar, B. L., \& Galinsky, A. D. (2016). Economic insecurity increases physical pain. Psychological, 27(4), 443-454. https://doi.org/10.1177/0956797615625640 
Edwards, W., Lindman, H., \& Savage, L. J. (1963). Bayesian statistical inference for psychological research. Psychological Review, 70(3), 193-242.

http://dx.doi.org/10.1037/h0038674

Edwards, A. W. F. (1972). Likelihood. Cambridge: Cambridge University Press (expanded edition, 1992, Johns Hopkins University Press, Baltimore).

Faul, F., Erdfelder, E., Lang, A. G., \& Buchner, A. (2007). G* Power 3: A flexible statistical power analysis program for the social, behavioral, and biomedical sciences. Behavior research methods, 39(2), 175-191.

https://doi.org/10.3758/BF03193146

Fisher, R.A. (1956). Statistical methods and scientific inference. New York: Hafner.

Francis, G. (2012). The psychology of replication and replication in psychology.

Perspectives on Psychological Science, 7, 585-594.

https://doi.org/10.1177/1745691612459520

Gigerenzer, G. (2018). Statistical rituals: The replication delusion and how we got there. Advances in Methods and Practices in Psychological Science, 1(2), $198-218$.

Gervais, W. M., Jewell, J. A., Najle, M. B., \& Ng, B. K. L. (2015). A powerful nudge? Presenting calculable consequences of underpowered research shifts incentives toward adequately powered designs. Social Psychological and Personal Science, 6(7), 847-854. https://doi.org/10.1177/1948550615584199

Hagger, M. S., \& Chatzisarantis, N. L. D. (2016). A multilab preregistered replication of the ego-depletion effect. Perspectives on Psychological Science, 11(4), 546-573. https://doi.org/10.1177/1745691616652873

Ioannidis, J. (2005). Why most published research findings are false. PLoS medicine, 2, e124.

Kahneman, D. (2011). Thinking, fast and slow. New York: Farrar, Straus and Giroux.

Klein, R. A., Vianello, M., Hasselman, F., Adams, B. G., Adams, R. B., Jr., Alper, S., .., \& Nosek, B. A. (2018, November 21). Many Labs 2: Investigating Variation in Replicability Across Sample and Setting. Retrieved from http://osf.io/ux3eh

Krefeld-Schwalb, A.; Witte, E. H., \& Zenker, F. (2018). Hypothesis-testing demands trustworthy data - a simulation approach to statistical inference 
advocating the research program strategy. Frontiers in Psychology, 9, 460.

https://doi.org/10.3389/fpsyg.2018.00460

Lakatos, I. (1978). The Methodology of Scientific Research Programmes (ed. J.

Worrall and G. Currie). Cambridge: Cambridge University Press.

Landy, J. F., Jia, M. L., Ding, I. L., Viganola, D., Tierney, W., Dreber, A., ... \& Ly, A. (2020). Crowdsourcing hypothesis tests: Making transparent how design choices shape research results. Psychological Bulletin (online first, 13 April 2020).

McGrath, R. E., \& Meyer, G. J. (2006). When effect sizes disagree: The case of $r$ and d. Psychological Methods, 11(4), 386-401. https://doi.org/10.1037/1082$\underline{989 X .114 .386}$

Meehl, P. E. (1967). Theory testing in psychology and physics: A methodological paradox. Philosophy of Science, 34, 103-115. https://doi.org/10.1086/288135

Meehl, P. E. (1978). Theoretical risks and tabular asterisks: Sir Karl, Sir Ronald, and the slow progress of soft psychology. Journal of Consulting and Clinical Psychology, 46(4), 806-834.

Mayo, D. G. (1996). Error and the growth of experimental knowledge. Chicago: University of Chicago Press.

National Academies of Sciences, Engineering, and Medicine (2019). Reproducibility and Replicability in Science. Washington, DC: The National Academies Press. https://www.nap.edu/catalog/25303/reproducibility-and-replicability-in$\underline{\text { science }}$

Neyman, J., \& Pearson, E.S. (1967). Joint statistical papers. Cambridge: Cambridge University Press.

Nosek, B. A., \& Bar-Anan, Y. (2012). Scientific utopia I. Opening scientific communication. Psychological Inquiry, 23, 217-243. https://doi.org/10.1080/1047840X.2012.692215

Nosek, B. A.; Spies, J. R., \& Motyl, M. (2012). Scientific utopia: II. Restructuring incentives and practices to promote truth over publishability. Perspectives on Psychological Science, 7(6), 615-631. https://doi.org/10.1177\%2F1745691612459058

Open Science Collaboration (2015). Estimating the reproducibility of psychological science. Science, 349 (6251), acc4716-1-8. https://doi.org/10.1126/science.aac4716 
Pashler, H., \& Wagenmakers, E. J. (2012). Editors' introduction to the special section on replicability in psychological science: A crisis of confidence? Perspectives on Psychological Science, 7(6), 528-530. https://doi.org/10.1177/1745691612465253

Preacher, K. J., \& Kelley, K. (2011). Effect Size Measures for Mediation Models: Quantitative Strategies for Communicating Indirect Effects. Psychological Methods, 16(2), 93-115. http://dx.doi.org/10.1037/a0022658

Rehg, W. (2009). Cogent Science in Context. The Science Wars, Argumentation Theory and Habermas. Cambridge, MA: The MIT Press.

Schäfer, Th. \& Schwarz, M.A. (2019). The meaningfulness of effect sizes in psychological research: Differences between sub-disciplines and the impact of potential biases. Frontiers in Psychology, 10, 813. https://doi.org/10.3389/fpsyg.2019.00813

Schimmack, U., Moritz, H., \& Kesavan, K. (2017). Reconstruction of a Train Wreck: How Priming Research Went Off the Rails. Retrieved March 20, 2020 from https://replicationindex.com/2017/02/02/reconstruction-of-a-trainwreck-how-priming-research-went-of-the-rails/comment-page-1/\#comments (Comment by D. Kahman, posted February 14, 2017 at 8:37 pm).

Schönbrodt, F., \& Wagenmakers, E.-J. (2018). Bayes factor design analysis: Planning for compelling evidence. Psychonomic Bulletin \& Review, 25, 128142. http://dx.doi.org/10.3758/s13423-017-1230-y

Schulze, R. (2004). Meta-Analysis. A comparison of approaches. Göttingen: Hogrefe \& Huber.

Sedlmeier, P., \& Gigerenzer, G. (1989). Do studies of statistical power have an effect on the power of studies? Psychological Bulletin, 105, 309-316. http://dx.doi.org/10.1037/10109-032

Serra-Garcia, M., \& Gneezy, U. (2021). Nonreplicable publications are cited more than replicable ones. Science advances, 7(21), eabd1705. https://www.science.org/doi/10.1126/sciadv.abd1705

Stanley T. D., Carter, E. C., \& Doucouliagos, H. (2018). What meta-analyses reveal about the replicability of psychological research. Psychological Bulletin, 144 (12), 1325-1346. http://dx.doi.org/10.1037/bul0000169

Wald, A. (1947). Sequential Analysis. New York: Wiley. 
Wetzels, R., Matzke, D., Lee, M. D., Rouder, J. N., Iverson, G. J., \& Wagenmakers, E.-J. (2011). Statistical evidence in experimental psychology: An empirical comparison using $855 \mathrm{t}$ tests. Perspectives on Psychological Science, 6(3), 291-298. http://dx.doi.org/10.1177/1745691611406923

Witte, E. H. (1994). A statistical inference strategy (FOSTIS): A non-confounded hybrid theory. HAFOS, 9. http://hdl.handle.net/20.500.11780/491

Witte, E. H., \& Kaufman, J. (1997). The stepwise hybrid statistical inference strategy: FOSTIS. HAFOS, 18. http://hdl.handle.net/20.500.11780/502

Witte, E. H., \& Strohmeier, C. E. (2013). Forschung in der Psychologie. Ihre disziplinäre Matrix im Vergleich zu Physik, Biologie und Sozialwissenschaft. Psychologische Rundschau, 64, 16-24. https://doi.org/10.1026/0033-3042/a0000145

Witte, E. H., \& Zenker, F. (2016). Reconstructing recent work on macro-social stress as a research program. Basic and Applied Social Psychology, 38(6), 301-307. https://doi.org/10.1080/01973533.2016.1207077

Witte, E. H., \& Zenker, F. (2017a). Extending a multilab preregistered replication of the ego-depletion effect to a research program. Basic and Applied Social Psychology, 39(1), 74-80. https://doi.org/10.1080/01973533.2016.1269286

Witte, E. H., \& Zenker, F. (2017b). From discovery to justification. Outline of an ideal research program in empirical psychology. Frontiers in Psychology, 8, 1847. https://doi.org/10.3389/fpsyg.2017.01847

Witte, E. H., \& Zenker, F. (2018). Data replication matters, replicated hypothesiscorroboration counts. (Commentary on "Making Replication Mainstream" by Rolf A. Zwaan, Alexander Etz, Richard E., Lucas, and M. Brent Donnellan). Behavioral and Brain Sciences, 41, e120. https://doi.org/10.1017/S0140525X18000924

Yarkoni, T. (2020). The generalizability crisis. Behavioral and Brain Sciences, 1-37 (online first). https://doi.org/10.1017/S0140525X20001685

\section{Word count: 8886}

